Supplement Issue 1: International Tehran Breast Cancer Congress (TBCC9)

\title{
Influence of BDNF Genetic Variation on Breast Cancer Pain Severity Following Surgery
}

\author{
Mashayekhi Sohail ${ }^{1, *}$, Hamid Saeidi Saedi ${ }^{2}$ \\ ${ }^{1}$ Student Research Committee, Guilan University of Medical Sciences, Rasht, Iran \\ ${ }^{2}$ Department of Radiation Oncology, Cancer Research Center, Guilan University of \\ Medical Sciences, Rasht, Iran \\ ${ }^{*}$ Corresponding author: Mashayekhi Sohail, Student Research Committee, Guilan \\ University of Medical Sciences, Rasht, Iran.E-mail:Smashayekhi71@yahoo.com
}

DOI: $10.21859 /$ mci-supp-109

\author{
Keywords: \\ BDNF \\ Breast Cancer \\ Pain \\ Genetic Polymorphism
}

\begin{abstract}
Introduction: The genetics of chronic post-surgical pain have been largely demonstrated in recent years. Brain-derived neurotrophic factor (BDNF), as a member of the neurotrophin family of growth factors, plays neurotrophic and other physiological roles. The secretion of precursor and mature BDNF protein is affected by a single-nucleotide polymorphism (SNP) rs6265 (Val66Met, G196A) in the coding region of exon V of the BDNF gene. Genetic variability in the BDNF gene (Val66Met) may contribute to individual differences in cancer pain severity after surgery.

Materials and Methods: 350 patients were enrolled prior to and followed for six months after breast cancer surgery. 150 cases reported postsurgical pain at the wound site. The BDNF polymorphism was genotyped by PCR-RFLP. All statistical analyses were conducted using the MedCalc software (version 12.1).

Results: Genotype frequency of BDNF polymorphism showed significant difference in two groups. Individuals carrying the AA genotype were 3.8-fold at a higher risk of cancer pain severity compared with GG genotype ( $\mathrm{OR}=3.89$; 95\% CI, 2.02-7.49; $\mathrm{P}<0.001)$. Also, heterozygous carriers $(\mathrm{AG})$ have a higher risk of pain $(\mathrm{OR}=2.11$; 95\% CI, $1.23-$ 3.63; $\mathrm{P}=0.006)$.

Conclusions: Focusing on BDNF genotypic structure may help to understand the pathophysiology of pain perception and to identify therapeutic targets and biomarkers for pain in patients with breast cancer after surgery.
\end{abstract}

